\title{
Mirar la diversidad cultural para interculturalizar la educación superior
}

\author{
Looking at Cultural Diversity to interculturalize Higher Education
}

\author{
Marcia Mandepora Chundary'
}

\section{Resumen}

Lo habitual es que, en la educación superior universitaria, la interculturalidad es entendida como sinónimo del folklor, sinónimo de indígena y su acción se reduce al ámbito funcional o romántico. Si bien algunas universidades muestran ciertos avances a partir de la folklorización de las culturas y las lenguas; sin embargo, estos esfuerzos iniciales no inciden en las transformaciones curriculares, lo que significa que se sigue anulando la presencia de la diversidad de conocimientos y saberes ancestrales en estos espacios académicos.

Desde los pueblos indígenas la interculturalidad denota claramente su contenido histórico político, es decir, tiene una connotación altamente emancipatoria que plantea la superación de sistemas de dominación colonial y ejercicio de sus derechos colectivos e individuales. Este carácter histórico y político de la interculturalidad planteado por los pueblos indígenas no es comprendida a cabalidad, por eso las tendencias de folklorizar en el abordaje desde lo étnico, y en muchos casos desde el pretexto de que "todos somos iguales" se fortalece las políticas de inclusión y sometimiento.

Desde estas concepciones es que en este ensayo se reflexiona sobre las diversas formas de comprender la interculturalidad y su implementación, por lo cual asumo que, es necesario aportar con elementos de discusión para crear condiciones de avance hacia la interculturalización de la educación superior.

Palabras clave: Educación Superior; interculturalidad; pueblos indígenas.

\section{Abstract}

In higher education, interculturality is understood as a synonym for folklore, a synonym for indigenous, and its action is reduced to the functional or romantic sphere. Although, some universities show certain advances from the folklorization of cultures and languages; nevertheless, these initial efforts do not affect curricular transformations, which means that the presence of the diversity of ancestral knowledge and knowings in these academic spaces continues to be disregarded.

From indigenous peoples, interculturality clearly denotes its political historical content, that is, it has a highly emancipatory connotation that poses the overcoming of systems of colonial domination and the exercise of their collective and individual rights. This historical and political nature of the interculturality raised by indigenous peoples is not fully understood, which is why the tendencies of folklorizing in the ethnic approach, and in many cases from the pretext that "we are all equal", policies of inclusion and submission are strengthened.

\footnotetext{
1 Magíster en Educación Intercultural Bilingüe con Mención en Gestión y Planificación. Directora Ejecutiva FUNPROEIB Andes- Cochabamba Bolivia. Email: araendi05@gmail.com. DOI: https://orcid.org/0000-0001-7172-7440 
From these conceptions it is that this essay reflects on the different ways of understanding interculturality and its implementation, so it is assumed that it is necessary to contribute with elements of discussion to create conditions for progress towards the interculturalization of higher education.

Keywords: Higher Education; interculturality; indigenous peoples.

\section{Introducción}

Este ensayo presenta algunas reflexiones sobre la concepción de la interculturalidad, así como la importancia de su puesta en práctica para interculturalizar la educación superior y las situaciones conflictivas generadas a partir de la aplicación de esta. En este sentido, Mato (2018: 7) manifiesta que "la realidad de los pueblos indígenas en el mundo, todavía están sujetas a diversas formas de exclusión y discriminación en los sistemas educativos formales, incluyendo la educación superior".

Frente a la situación de exclusión, los pueblos indígenas demandan una educación con pertinencia cultural, acorde a su filosofía de vida y con enfoque intercultural, los cuales suponen la superación del neocolonialismo vigente. En este sentido, se debe entender que los lineamientos y fundamentos políticos de la educación no sólo deben referirse a una transformación de la práctica pedagógica, sino que fundamentalmente debe tener un contenido político e ideológico que permita a los pueblos indígenas plantear sus reivindicaciones, lograr una mayor participación, ejercer y acceder a diversas instancias de toma de decisiones y del poder, donde la educación se constituye como instrumento fundamental para los pueblos indígenas.

La finalidad de la presente reflexión es contribuir al proceso de debate sobre la interculturalidad en los espacios académicos universitarios, lograr que poco a poco reconozcan y tomen importancia a la diversidad cultural y lingüística al interior de las mismas universidades.

\section{Desarrollo}

\section{Diversas formas de comprender la interculturalidad}

En muchas situaciones, cuando se habla de la interculturalidad lo más habitual es relacionar este concepto con manifestaciones culturales de algún grupo étnico. Las actividades comunes que se observan en los establecimientos de educación regular es la feria de comidas tradicionales, artesanías, festival de música originaria, danzas, concurso de poesías y canto en lenguas indígenas. Asimismo, en algunos escenarios académicos de educación superior se comienza a promover este tipo de actividades, aunque con menos frecuencia o en facultades de ciencias humanas y sociales, solamente.

La carrera de Antropología de la Universidad Mayor de San Simón², por las festividades de Todos Santos ha realizado festival intercultural del mast 'aku aymarq'ay killa que en castellano significa (armado de la mesa para el mes de los muertos), en la cual participaron estudiantes de colegios y de la misma universidad. Esta actividad se puede considerar como muy buena iniciativa de los docentes para difundir las culturas originarias a los estudiantes universitarios y docentes, ya que en estos espacios académicos se encuentran estudiantes de diversas culturas, y es positivo para que empiecen a valorar sus culturas en estos espacios, es un buen inicio para avanzar hacia la interculturalización de la educación superior.

2 La universidad Mayor de San Simón es parte sistema de universidades públicas convencionales. 
Las actividades de carácter cultural en los escenarios académicos de educación superior son desarrolladas por profesionales formados en interculturalidad. Los profesionales que organizaron la actividad mencionada anteriormente tienen formación en interculturalidad y experiencias de trabajos con pueblos indígenas, uno de ellos es egresado de la Maestría en Educación Intercultural Bilingüe del PROEIB Andes, y otro, es el ex - Rector de la Universidad Quechua Casimiro Huanca ${ }^{3}$, ambos, docentes de la Carrera de Antropología. A partir de lo señalado, se puede inferir el origen de la promoción de las actividades en espacios académicos de la universidad que permite la sensibilización hacia las diversas culturas.

Si bien en el texto de Mato (2018) señala que, los programas de inclusión como estudiantes en universidades convencionales promueve "fuga de cerebros" desde las comunidades, logrando su permanencia en las ciudades; sin embargo muchos profesionales indígenas logran resistir e imponer su cultura, su lengua, sus valores étnicos, sus conocimientos, saberes y prácticas ancestrales, aun batallando con problemas de racismo y discriminación; aunque los programas se limitan a garantizar el acceso a la educación superior las becas; pero, estas le abren la posibilidad de incidir en diversos campos tanto desde la docencia y la vinculación de las comunidades con las universidades.

En la Facultad Integral del $\mathrm{Chaco}^{4}$ de la Ciudad de Camiri, en la malla curricular de la carrera de Ingeniería Socio Ambiental se ha incorporado la materia de lengua originaria guaraní y la materia de conocimientos indígenas en actividades productivas, desde estas asignaturas se enseña el idioma guaraní, se aborda la cultura guaraní y de otros pueblos indígenas de Bolivia. De la misma manera en la malla curricular de la Carrera de Ciencias de la Educación se incorpora la materia de Fundamento de la Educación Intercultural Bilingüe, desde esta asignatura se aborda los hitos históricos que dan inicio a la educación con pertinencia cultural y lingüística y su incorporación como política pública del Estado.

La incorporación formal en la malla curricular de los temas relacionados a pueblos indígenas, si bien no es suficiente, considero como un desafío y una forma efectiva y formal de interculturalizar la educación superior, porque es una posibilidad de iniciar reflexiones teóricas desde las aulas universitarias y lograr posicionar la temática indígena en los escenarios académicos y no se sigan entendiendo como sinónimo de folklor, más aún cuando en esta casa superior de estudios, provienen estudiantes de diversas culturas, queda la tarea de continuar incidiendo en la interculturalización de la educación superior en sus diferentes niveles y ámbitos.

La existencia de algunos indicios de avances de conocimiento de la diversidad cultural en los escenarios académicos de la educación superior, es por la presencia de profesionales docentes indígenas y profesionales no indígenas comprometidos con las causas y luchas indígenas, así como diría (Mato 2018), existen "docentes e investigadores que no se autoidentifican como miembros de pueblos indígenas, pero que participan en estas experiencias" son actores claves que contribuyen en gran medida desde las aulas y otros espacios en la sensibilización de los estudiantes, docentes y autoridades hacia la valoración de la diversidad cultural, aunque el desafío más importante es "promover la diversidad cultural y la interculturalidad en condiciones equitativas y mutuamente respetuosas" (Mato, 2018: 87).

Es importante reconocer a diversos actores, quienes han contribuido al proceso de la interculturalización de la educación y al proceso de inclusión de los indígenas en la educación superior; estos actores actuaron desde diferentes niveles, ámbitos, campos, experiencias, áreas, espacios territoriales, logrando establecer acuerdo institucionales con instituciones de educación superior y otras instituciones, principalmente con

3 La universidad Casimiro Huanca, es indígena e intercultural para la población quechua.

4 Es la unidad académica desconcentrada de la Universidad Autónoma de la Gabriel René Moreno de Santa Cruz, que pertenece al sistema de universidades públicas convencionales. 
la Cooperación Internacional para garantizar el acceso y la permanencia de indígenas en la educación superior, aunque de carácter convencional.

En la interculturalidad la palabra clave es el diálogo. La interculturalidad reasume dimensiones decisivas del multiculturalismo, en el sentido de que para dialogar hay que presuponer respeto mutuo y condiciones de igualdad entre quienes dialogan, lo que implica que desde la interculturalidad se propugna el diálogo y encuentro entre culturas como expresión de solidaridad entre ellas. Sin embargo, la interculturalidad no precisa sólo las condiciones de respeto mutuo y de igualdad de circunstancias sociales, precisa también que los grupos implicados reconozcan recíprocamente su capacidad de creación cultural. Entonces, la interculturalidad es un modo específico de autoafirmación etno identitaria, afirmación de los derechos culturales; implica que la asunción de la pluriculturalidad es la base y la condición de posibilidad de la interculturalidad.

Los movimientos indígenas desde su proyecto político plantean el concepto de interculturalidad como una transformación, no sólo de las actitudes coloniales, en un Estado que todavía reproduce la ideología del colonialismo interno, sino que principalmente reclama la necesidad de que el Estado permita a los pueblos indígenas ser parte activa del ejercicio de sus derechos con todas las diferencias de carácter ético, político y epistémicos que esto conlleva. El proyecto de interculturalidad reconoce las diferencias como y una potencialidad y no como un obstáculo, también como espacio de participación e intervención de los excluidos en igualdad de condiciones; razón por la que la interculturalidad en muchos países se convierte en política de Estado (López \& Küper, 2003).

El concepto de la interculturalidad ha comenzado a trascender la esfera indígena para impregnar con sus ideas, propuestas y utopías al conjunto de la educación y la sociedad. Sin embargo, las propuestas de interculturalidad, siguen ancladas en el plano discursivo y difícilmente influyen suficientemente en la vida cotidiana de la gente; y menos se modifican las relaciones sociales entre ellas. En muchos casos, desde las voces de los propios dirigentes indígenas se escucha decir que, los indígenas siempre fueron interculturales, porque siempre vivieron en armonía. Sin embargo, en algunos casos, se observan situaciones conflictivas entre los pueblos indígenas; y los conflictos se dan por la ocupación de la tierra, distribución de los recursos económicos, constante migración de los indígenas a otros pueblos y ciudades, y también por el rechazo de otras culturas sean indígenas o no indígenas, toda esta situación es muestra de la existencia de la desigualdad social entre las culturas indígenas.

Habitualmente la interculturalidad se aborda desde la dimensión étnica, sin tomar en cuenta otras dimensiones de la diversidad de un contexto. Razones sobran, conocemos que los pueblos indígenas son los que han sido avasallados, expropiando sus culturas, lenguas, entonces por mucho tiempo han desvalorado su propia cultura, por lo tanto, era necesario volver a concientizar al propio indígena para que vuelva a valorar su cultura, lengua, conocimientos y saberes y recuperar o fortalecer su autoestima. Probablemente fue una de las justificaciones para que en un inicio la educación intercultural bilingüe se desarrollara para los pueblos indígenas y no así para la sociedad en su conjunto5 , pero con énfasis en el desarrollo del bilingüismo, más que la interculturalidad.

En suma, se puede decir que la interculturalidad inicialmente fue un concepto dirigido a los miembros de pueblos, culturas y lenguas originarias y orientadas a contribuir a que éstos recuperen la confianza perdida respecto de sí mismos, de sus pueblos y de sus instituciones culturales y lingüísticas.

Las experiencias han demostrado que la vulneración de los derechos de los pueblos indígenas provenía generalmente de la sociedad no indígena, por lo que era necesario extender el trabajo de sensibilización y

5 En el caso boliviano con la Ley 1565 de la Reforma Educativa se implementó la EIB para algunos pueblos indígenas. 
lograr el reconocimiento de los derechos de los pueblos indígenas por la sociedad no indígena a través de la política de la generalización de la Educación Intercultural Bilingüe para toda la sociedad.

Cabe recordar que la educación fue y es considerada herramienta principal que plantean los pueblos indígenas para la construcción de sociedades interculturales sustentadas en la riqueza de la diversidad, así se plantea que si la educación intercultural no asumiera desde la praxis la diversidad cultural, se quedará en buen intento parecido a muchos otros programas, cuyo único resultado es la asimilación de la cultura de las minorías étnicas a la cultura nacional y dominante.

La interculturalidad aplicada a la educación, en la opinión de Walter Gutiérrez, ex presidente del Consejo Educativo Aimara es que hay "una interculturalidad entre pueblos originarios, que es intercultural entre iguales, y en otras que es desigual y se da entre los pueblos indígenas y los de la sociedad dominante. La interculturalidad tiene que fortalecer la unidad de los pueblos indígenas hacia adentro y tiene que servir como lucha para conseguir el respeto hacia fuera. Cuando hablamos de interculturalidad hay que ver que haya equilibrio (igualdad) tanto en los idiomas, en la religión, en la ciencia, en la filosofía y otros. Tiene que haber primero una igualdad en la dignidad de los que van a practicar la interculturalidad. Esta como herramienta política de las comunidades originarias hacia el Estado de la sociedad dominante". (Miranda, 2003, p. 36).

A partir de las diversas ideas y opiniones, existe la necesidad de darle sentido y contenido al concepto de la interculturalidad según cada contexto histórico y político de los pueblos indígenas, desde los cuales, la interculturalidad se entiende como emancipación, ejercicio de sus derechos y reconstitución de sus territorios ancestrales y espacio de fortalecimiento de sus culturas, lenguas, saberes y prácticas ancestrales, superando la visión reduccionista de que la interculturalidad es respeto, diálogo, tolerancia, armonía. Teniendo precaución de que desde estos enunciados se puede vulnerar los derechos individuales y colectivos de los pueblos indígenas, justificando que todos somos iguales.

\section{Mirar a la diversidad cultural para interculturalizar la educación superior}

Hablar de la interculturalidad en la educación superior, no se trata simplemente hablar de la presencia de la diversidad cultural, de la presencia de estudiantes de origen indígena, referirse al folklor, tampoco reducir a la enseñanza de lenguas indígenas, en la que probablemente no se dan mutuos intercambios o se están ocultando diferencias, desigualdades, marginación, racismo, donde existen la imposición de modelos educativos homogeneizantes y donde se da la "negación del valor de los conocimientos, lenguas, modos de producción de conocimiento y modalidades de aprendizaje, valores, visiones de mundo, historias y propuestas de los pueblos indígenas y afrodescendientes de América Latina" (Mato, 2018, p. 103).

Se trata de que las universidades presten atención a los estudiantes que provienen de pueblos indígenas para lo cual deben contar con datos estadísticos actualizados de estudiantes de origen o hablantes indígenas. Principalmente asumir el reto de revisar, adecuar, elaborar el currículo con enfoque intercultural donde se incorporen como asignaturas y contenidos, conocimientos, saberes y prácticas ancestrales de los pueblos indígenas y afrodescendientes. De esta manera es posible garantizar la construcción de una pedagogía intercultural con base en la diversidad, una pedagogía que procure formar sujetos que sean capaces de vivir y convivir en un contexto multicultural.

Para promover la interculturalidad en la educación superior, es necesario contar con docentes capacitados, convencidos y comprometidos con el trabajo, de esta manera es posible generar condiciones, como muchos dicen, hacer el trabajo desde "abajo" con diferentes actores, desde las aulas, desde las prácticas pedagógicas, es decir avanzar desde el nivel discursivo a la práctica. Para lo cual se necesita que los profesionales tengan 
conocimientos sobre la interculturalidad, pero en realidad la mayoría de los docentes de las universidades no tienen conocimientos y menos desarrollan la interculturalidad.

Los docentes de la primaria y secundaria son los que mejor conocimientos tienen sobre el tema, porque están en permanente capacitación, actualización y profesionalización por parte del Ministerio de Educación, y por lo tanto son los que más reproducen la idea de que la interculturalidad es comunicación, relación con otras culturas, convivencia, respeto, tolerancia y aceptación de esas otras culturas, siguiendo con las orientaciones de la UNESCO (2002), que manifiesta atender el fenómeno de interculturalidad desde el respeto a las diferencias y su aceptación como un aporte a la creatividad humana y al manejo de conflictos para la convivencia y una cultura de cooperación y paz.

En los escenarios académicos de la educación superior muy poco se ha avanzado, aunque algunas universidades como parte de los principios y valores de su modelo educativo incorpora el concepto de la interculturalidad. La Universidad Mayor de San Simón de Cochabamba incorpora la interculturalidad en los siguientes términos: "la interrelación y convivencia equitativa, en igualdad de oportunidades para todas y todos, a través de la valoración y respeto recíproco entre culturas, el reconocimiento y valoración de las distintas cosmovisiones y el diálogo entre saberes distintos" (UMSS, 2014:37). De la misma manera como parte de sus fundamentos sociopolíticos asume la educación superior como intra e intercultural en concordancia con la política del Estado Plurinacional de Bolivia y la Ley Educativa 07o Avelino Siñani y Elizardo Pérez (UMSS, 2014, p. 41).

Aparentemente se establece como legado institucional, pero aún está lejos de ser aplicado porque se sigue conservando contenidos, metodologías de una educación conservadora tradicional y aún están lejos de reconocer la existencia de la diversidad cultural en su interior. Existe un discurso político pero que no se implementa en las aulas, que son los núcleos más duros de la educación superior, y mucho menos existe la intención de modificar los currículos, porque afecta el interés de los docentes en cuanto a su carga horaria, lo que menos pretenden las autoridades es crear conflictos a raíz de descontentos o disconformidad con el cambio, es decir, lo que prima es el interés de los docentes antes que los estudiantes.

Si bien se han desarrollado experiencias exitosas en el tema de la interculturalidad como parte de programas de formación a nivel de pregrado y postgrado; pero, estas experiencias no han sido asumidas y desarrolladas como parte constitutiva de la universidad, son programas que poco se vinculan y se manejan de manera aislada, por lo tanto, existe poca incidencia en la trasformación de las prácticas tradicionales. Esta es una muestra de la ausencia del compromiso y voluntades políticas de las autoridades académicas, para aplicar las normas a favor de la Interculturalización de la educación superior, y mucho menos contribuir a la Interculturalización de la sociedad en su conjunto.

Parece necesario acudir a una colaboración intercultural entre diferentes actores: instituciones, docentes, investigadores y estudiantes, en el marco de la confianza, actitudes positivas y predisposición para generar condiciones técnicas, políticas, "en la cual la colaboración intercultural se desarrolle mediante vinculación, extensión, servicio u otras denominaciones orientados a mejorar la calidad de vida de las comunidades". (Mato 2018).

Se requiere una transformación profunda que permita a las universidades reencontrarse con la realidad de los pueblos, y romper con su alineamiento histórico con una sola fuente de conocimientos y valores, para que imbuida del espíritu de la interculturalidad, recoloque su rol en la sociedad, repiense su misión y su manera de cumplir con su cometido. 
Valorar los avances logrados es muy importante, según Mato (2018), estos logros son resultados de las luchas sociales y políticas de los pueblos indígenas y sus organizaciones, así como de los movimientos sociales anti-racismo, de derechos humanos, como también, son resultados de las prácticas institucionales de algunas universidades y otros tipos de educación superior, así como a los de varias agencias intergubernamentales de cooperación internacional y diversidad de actores involucrados en el proceso. Sin embargo, el camino a andar es aún lejano, por las resistencias y falta de compromiso para asumir el reto de aplicar la interculturalidad.

La Conferencia Regional de Educación Superior CRES (2018), proclama la interculturalización de la educación superior en los siguientes términos:

Es necesario promover la diversidad cultural y la interculturalidad en condiciones equitativas y mutuamente respetuosas. El reto no es solo incluir en las instituciones de educación superior a mujeres, personas con discapacidad, miembros de pueblos indígenas y afrodescendientes e individuos de grupos sociales históricamente discriminados, sino transformarlas para que sean social y culturalmente pertinentes. Estos cambios deben asegurar la incorporación en las instituciones de educación superior de las cosmovisiones, valores, conocimientos, saberes, sistemas lingüísticos, formas de aprendizaje y modos de producción de conocimiento de dichos pueblos y grupos sociales. (CRES. 2018).

La educación superior intercultural se constituye en un desafío importante, así en algunos países de América Latina, como señala Mato (2018:4), "Las experiencias de Educación Superior Indígena se han desarrollado en el marco de diversos tipos de arreglos institucionales, en algunos casos se han hecho como programas especiales de universidades convencionales, o bien mediante alianzas entre estas últimas y organizaciones, como también se ha desarrollado en el marco de universidades creadas y gestionadas por dirigentes y/u organizaciones de pueblos indígenas y en el marco de universidades creadas por el Estado". También existen universidades indígenas generadas y creadas desde las propuestas educativas de los pueblos indígenas, tienen una base social y política, y tienen la posibilidad de diseñar su currículo, su gestión institucional en coherencia con su concepción filosófica.

Lo que se pretende con las Instituciones de Educación Superior con carácter intercultural es superar el sistema de discriminación, exclusión, asimilación y de racismo vigente.

\section{Conclusiones}

La transformación de las universidades es una necesidad fundamental, ya que debe impulsar el desarrollo social y la producción de saberes y conocimientos, abordando y asumiendo con una mirada, de contextualización y de interpretación de la realidad social.

El avanzar de la interculturalidad discursiva a la práctica es reto que se debe asumir; promover la investigación sobre conocimientos indígenas es una necesidad que se tiene en este momento; producir materiales didácticos en lenguas indígenas; formar docentes interculturales; capacitar y sensibilizar a los docentes que actualmente se encuentran trabajando.

Otro desafío importante, y el más difícil es el diálogo de saberes, aunque se está realizando en la medida de las posibilidades de las condiciones generadas en diferentes espacios educativos. Considero que es tarea de todos, el aprender a entablar diálogos, más que mostrar una oposición es aprender a establecer puntos de vistas interculturales, solamente de esta manera será posible recuperar, sistematizar experiencias diversas desarrolladas a nivel de Latinoamérica. 
Las universidades deben asumir responsabilidad institucional frente a sujetos negados. Atender a otras formas de saber y conocer y establecer el vínculo de la problemática y el proyecto nacional. Las universidades deben asumir la interculturalidad en todos sus niveles, ámbitos y sus estructuras académicas y administrativas. Las universidades deben generar información y datos estadísticos sobre la procedencia de la población estudiantil para definir políticas e interculturalizar la universidad. Las universidades deben recuperar, valorar, replicar y difundir las experiencias y las lecciones aprendidas de los proyectos y programas con y para universitarios indígenas implementados al interior de la propia universidad. Tomar la diversidad como eje de la construcción universitaria, desde la triada formación, investigación y práctica.

En suma, la universidad debe ser propiciadora y creadora de innovaciones propias, construir procesos pedagógicos que permitan un aprendizaje en la vida y para la vida, con una pedagogía comunitaria, productiva, participativa e ideológica, de manera que la convivencia armónica se convierta en un espacio de aprendizaje cooperativo y de reciprocidad, a través de la recuperación de experiencias, conocimientos y saberes diversos.

\section{Lista de referencias}

CRES. (2018). Sección del eje temático Educación Superior, Diversidad Cultural e Interculturalidad de la Declaración de la zra. Conferencia Regional de Educación Superior. Disponible en: http://www.cres2o18. org/uploads/declaracion_cres2018\%20(2).pdf

Delors, J. (2002). “La educación encierra un tesoro”. Madrid: Santillana-UNESCO.

López, L., \& Küper W. (2003). "La EIB en America Latina: Balance y perspectivas. PROEIB Andes. Cochabamba, Bolivia.

Mato, D. (2008). No hay saber "universal", la colaboración intercultural es imprescindible. Alteridades 18(35), 101-116.

Mato, D. (2016). Educación Superior y Pueblos Indígenas en América Latina: Del “Diálogo de Saberes” a la construcción de modalidades sostenibles de "Colaboración Intercultural". Revista del CISEN Tramas/ Maepova 4(2), 71-94.

Mato, D. (2018). Educación Superior y Pueblos Indígenas y Afrodescendientes en América Latina: Tendencias en curso, tensiones, posibilidades y desafíos. En: Pedro Henríquez Guajardo (coord.) Tendencias de la educación superior en América Latina y el Caribe 2018.

Ministerio de Educación y Culturas (2006). Nueva Ley de Educación “Avelino Siñani y Elizardo Pérez. La Paz: Ministerio de Educación

Miranda, T. (2003). “Documento de Sensibilización en EIB”. Tantanakuy, Cochabamba: PROEIB Andes.

Universidad Mayor de San Simon. (2014). Modelo Educativo. Cochabamba-Bolivia. 